\title{
KNOWLEDGE BASED ADAPTIVE PROCESSING FOR GROUND MOVING TARGET INDICATION
}

\author{
Raviraj Adve ${ }^{1}$, Todd Hale, and Michael Wicks
}

Raviraj Adve

Dept. of Elec. and Comp. Engg. University of Toronto

10 King's College Rd.

Toronto

Ontario M5S 3G4, Canada
Todd Hale

Air Force Inst. of Technology

2950 P Street

Wright-Patterson AFB

$\mathrm{OH} 45324$, USA

\author{
Michael Wicks \\ Air Force Research Laboratory \\ Sensors Directorate \\ 26 Electronics Parkway \\ Rome \\ NY 13403, USA
}

\begin{abstract}
This paper presents a preliminary knowledge based approach to Space-Time Adaptive Processing (STAP) for ground moving target indication from an airborne platform. The KB-processor accounts for practical aspects of adaptive processing, including detection and processing of non-homogeneous data, appropriate selection of training data, and accounting for array effects such as mutual coupling and channel mismatch. In combining these hitherto separate STAP issues into a unified approach, this paper furthers the move of STAP from theory to practice. The KB-approach is tested using measured data from the Multi-Channel Airborne Radar Measurements program.
\end{abstract}

\section{Introduction}

Space-Time Adaptive Processing (STAP) techniques promise to be the best means to detect weak targets in severe, dynamic, interference scenarios including clutter and jamming. STAP techniques were originally developed for airborne moving target indication (AMTI) [1,2], demonstrating superior interference rejection over non-adaptive techniques such as the displaced phased center array (DPCA) approach. STAP algorithms are only now being used for Ground Moving Target Indication (GMTI) from an air- or space-borne platform. Low velocity ground targets lie extremely close to the mainbeam clutter in Doppler, making detection difficult. Non-adaptive techniques are usually able to detect only large ground targets or must deal with high false alarm rates. The interference may also include broadband (barrage noise) or coherent jamming. The need to detect small, slow moving, ground targets in stressful interference environments drives research in adaptive processing techniques for GMTI.

The antenna array under consideration has $N$ equally spaced spatial channels. The array transmits a pulse in a chosen direction, the look angle. The returned signal is sampled rapidly $R$

\footnotetext{
${ }^{1}$ This work was performed while Prof. Adve was with Research Associates for Defense Conversion Inc., Marcy, NY. He was supported by the Air Force Research Laboratory under contract F-30602-97-C-0006.
} 
times with each sample corresponding to a range cell. Repeating this process $M$ times within a Coherent Processing Interval (CPI) results in a $N \times M \times R$ data cube. The array is attempting to detect a potential target within at specific range (the primary range cell) within this data cube. The most straightforward STAP algorithm uses all NM spatio-temporal degrees of freedom (DOF), estimating the $N M \times N M$ interference covariance matrix to minimize the mean squared error with respect to the desired signal [3]. The drawback is that an accurate estimate requires between $2 N M$ and $3 N M$ statistically homogeneous data samples [3], usually obtained from secondary data, i.e., range cells other than the primary range cell. Obtaining such a large number of homogeneous samples is usually impossible in practice. Furthermore, even if the required secondary data were available, the associated computation load makes this approach impractical for typical values of $N$ and $M$.

To overcome the drawbacks of the fully adaptive algorithm, researchers have developed reduced DOF techniques with corresponding reductions in computation load and required sample support [1]. However, the requirement of homogeneous secondary data remains. In practice, the interference is invariably non-homogeneous due to terrain variations, corner reflectors, etc. Another issue of practical importance is the use of physical antenna arrays. STAP algorithms were originally developed for proof of concept, assuming an ideal linear array of equispaced, isotropic, point sensors and ignoring real world effects such as mutual coupling and channel mismatch.

Traditional STAP algorithms suffer from significant performance loss in realistic scenarios that include non-homogeneous data and antenna array effects. This paper discusses the impact of these issues individually and then brings these diverse concepts together into a unified approach to STAP. The goal is to develop a knowledge based STAP (KB-STAP) approach that tailors the processing technique to the interference scenario at hand while accounting for practical considerations such as discrete non-homogeneities and mutual coupling between antenna elements. The results of these individual investigations have been published elsewhere [4-6]. The key contribution here is bringing these individual aspects together into a single, practical, KB-processor. The examples, using measured data, present the resulting performance enhancements and illustrate the potential of adaptive processing in real world scenarios for GMTI.

Recently, there has been growing agreement that effective adaptive processing for weak target detection requires a knowledge based approach [7-11]. These activities have generally been inspired by the DARPA Knowledge-Aided Sensor Signal Processing and Expert Reasoning (KASSPER) program [7]. These papers are generally focused on dealing with non-homogeneous clutter [8-11]. This paper addresses the issue, but also accounts for other real-world issues such as mutual coupling. It also introduces the use of hybrid processing specifically for range cells deemed non-homogeneous. In this regard, this paper develops a preliminary, but comprehensive, approach to KB-STAP.

The paper is organized as follows. Section 2 presents the various components of the KB-STAP 


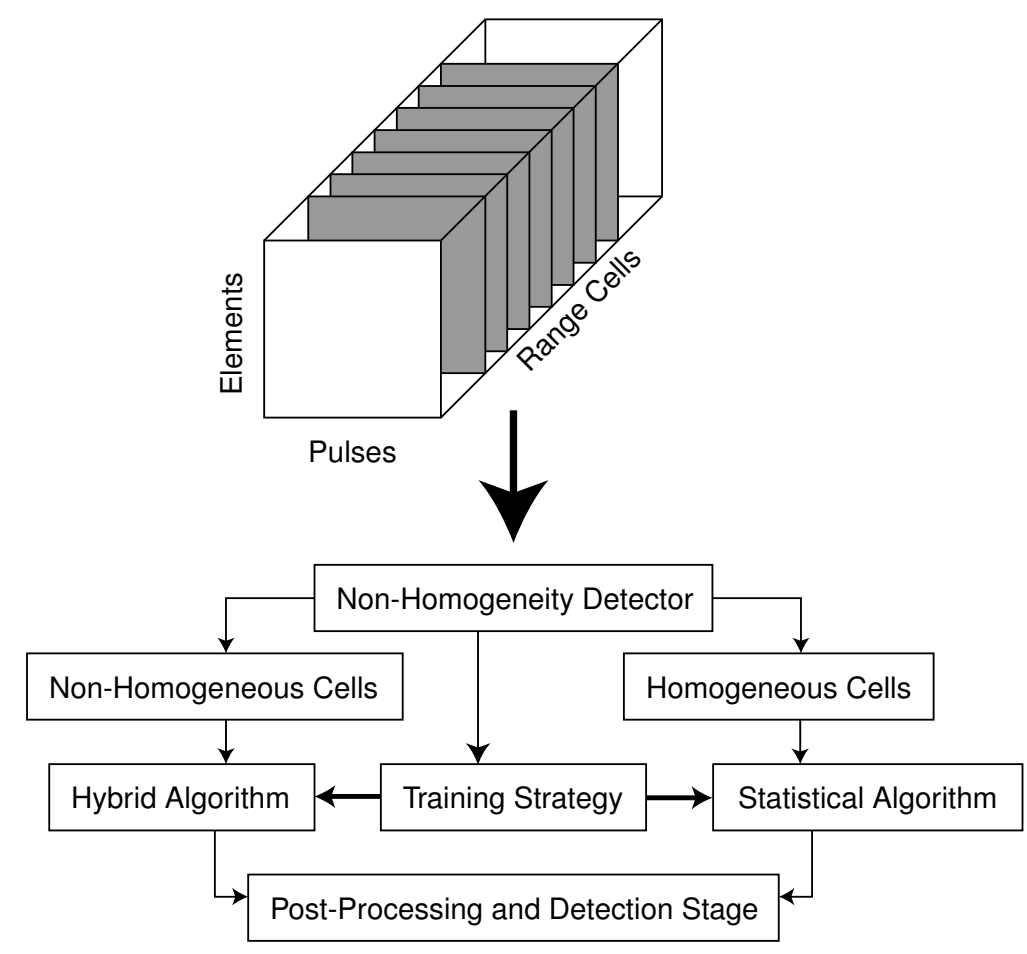

Figure 1: Knowledge Based Space-Time Adaptive Processing.

processor as described above. These components have been presented elsewhere and are included here for completeness [4-6]. The key contribution of this paper is presented in Section 3 where these components are combined into the KB-STAP processor described by Fig. 1. The performance of the KB-processor is tested using measured data from the Multi-Channel Airborne Radar Measurements (MCARM) program [12].

\section{Knowledge Based Processing}

Fig. 1 presents the elements of the KB-STAP processor, proposed here as an alternative to other proposed knowledge aided processors, such as in [8,9]. A common element to all these schemes is the use of processing best matched to the data and interference scenario at hand. The first step to any such processor must be an identification of the non-homogeneous data within the radar data cube, i.e., the first step is a Non-Homogeneity Detector (NHD). The NHD separates the range cells into homogeneous and non-homogeneous sections. Another common theme to all proposed KB-processors is the use of only homogeneous range cells in estimating interference statistics. However, the NHD used here focuses on only identifying those non-homogeneities that impact on the performance of the STAP algorithm under consideration.

A crucial distinguishing feature of the KB-structure proposed here is the use of a partially nonstatistical algorithm specifically for adaptive processing within range cells deemed non-homogeneous. 
Within these cells, correlated and discrete interference hinders target detection. For example, the interference may include fairly homogeneous ground clutter (which is correlated) and returns from a corner reflector (which is localized or discrete). Purely statistical algorithms are inappropriate because the data from surrounding range cells do not possess information about discretes. This paper uses the two-stage hybrid algorithm [6] with a non-statistical, yet adaptive, first stage to suppress the discrete interference component. This is followed by a second stage of statistical processing to suppress residual correlated interference.

Though not explicitly indicated in Fig. 1, a crucial requirement for practical adaptive processing is the need to account for effects associated with using practical antenna arrays. The analysis and compensation procedure is presented here in the context of the Joint Domain Localized (JDL) processing algorithm, the basis of all signal processing undertaken in Fig. 1. The results show that in dealing with measured data, significant performance gains can be achieved by utilizing measured antenna characteristics.

\subsection{Components of Proposed Knowledge-Based Processor}

This section details the components of the KB-STAP processor of Fig. 1. Section 2.2 presents a reformulation of the JDL algorithm for practical antenna arrays. Section 2.3 presents a nonhomogeneity detector based on the principle that only those non-homogeneities which explicitly impact STAP performance need be accounted for. The use of a transformation matrix, introduced in Section 2.2 to deal with practical arrays, plays a crucial role in the development of the hybrid algorithm for non-homogeneous data, presented in Section 2.4. We begin with a brief review of the fully adaptive processor for an ideal linear array.

Starting with the $N \times M \times R$ data cube, for each range bin, the received data can be written as a $N \times M$ matrix $\mathbf{X}$ or a length- $M N$ vector $\mathbf{x}$ whose entries numbered $m N$ to $[(m+1) N-1]$ correspond to the returns at the $N$ elements from pulse number $m, m=0,1, \ldots, M-1$. The data vector is a sum of the interference, thermal noise, and possibly a target,

$$
\mathbf{x}=\xi \mathbf{v}\left(\phi_{t}, f_{t}\right)+\mathbf{c}+\mathbf{n},
$$

where $\mathbf{c}$ represents the interference sources (both clutter and jamming), $\mathbf{n}$ the thermal noise, and $\xi$ is the target amplitude, equal to zero in range cells without a target. The term $\mathbf{v}\left(\phi_{t}, f_{t}\right)$ is the space-time steering vector describing the response of the array to a possible target at look angle $\phi_{t}$ (referenced to broadside) and Doppler frequency $f_{t}$. Note that in STAP, the steering vector only sets the look direction. In practice, there may be some beam mismatch between the true location of the target and the look steering vector. This steering vector can be written in terms of a spatial 
steering vector $\mathbf{a}\left(\phi_{t}\right)$ and a temporal steering vector $\mathbf{b}\left(f_{t}\right)[1]$,

$$
\begin{aligned}
\mathbf{v}\left(\phi_{t}, f_{t}\right) & =\mathbf{b}\left(f_{t}\right) \otimes \mathbf{a}\left(\phi_{t}\right), \\
\mathbf{a}\left(\phi_{t}\right) & =\left[\begin{array}{lllll}
1 & z_{s} & z_{s}^{2} & \ldots & z_{s}^{(N-1)}
\end{array}\right]^{T}, \\
\mathbf{b}\left(f_{t}\right) & =\left[\begin{array}{lllll}
1 & z_{t} & z_{t}^{2} & \ldots & z_{t}^{(M-1)}
\end{array}\right]^{T}, \\
z_{s} & =e^{j 2 \pi f_{s}}=e^{\left(j 2 \pi \frac{d}{\lambda} \sin \phi_{t}\right)}, \\
z_{t} & =e^{j 2 \pi f_{t} / f_{R}},
\end{aligned}
$$

where $\otimes$ represents the Kronecker product of two vectors, $f_{R}$ the pulse repetition frequency (PRF), and $\lambda$ the wavelength of operation. Note that this form of the spatial steering vector is valid only for a linear, equispaced array of isotropic sensors. The fully adaptive processor determines a set of weights $\mathbf{w}$ by solving the matrix equation [3]

$$
\begin{aligned}
\hat{\mathbf{R}} \mathbf{w} & =\mathbf{v}\left(\phi_{t}, f_{t}\right), \\
\hat{\mathbf{R}} & =\frac{1}{K} \sum_{k=1}^{K} \mathbf{x}_{k} \mathbf{x}_{k}^{H},
\end{aligned}
$$

where $\mathbf{x}_{k}$ represents one of $K$ secondary, target-free, data samples and ${ }^{H}$ represents the Hermitian (conjugate transpose) of a matrix. These weights are used to obtain a decision statistic to decide whether a target is present at that range bin or not. This paper uses a constant false alarm rate (CFAR) modified sample matrix inversion (MSMI) statistic [13],

$$
\rho_{\mathrm{MSMI}}=\frac{\left|\mathbf{w}^{H} \mathbf{x}\right|^{2}}{\mathbf{w}^{H} \mathbf{v}} .
$$

\subsection{JDL for practical arrays}

The JDL algorithm is a popular and effective STAP algorithm, combining the benefits of extremely low DOF with maximal gain against thermal noise. The first step in the original JDL algorithm is a transformation of the space-time data to the angle-Doppler domain. Adaptive processing is then performed within a localized processing region (LPR) comprising $\eta_{a}$ angle bins and $\eta_{d}$ Doppler bins [14]. This LPR is usually, though not necessarily, centered about the angle-Doppler look direction. The DOF is reduced to the size of the LPR $\left(\eta_{d} \eta_{a}\right)$, significantly reducing the required secondary data and associated computation load.

The spatial steering vector $\mathbf{a}(\phi)$ is the magnitude and phase taper received at the $N$ elements of the array due to a far field source at angle $\phi$. Due to electromagnetic reciprocity, to transmit in the direction $\phi$ the elements of the array must be excited with the conjugates of the steering vector, i.e., the conjugates of the steering vector maximize the response to the direction $\phi$. Transformation 
of spatial data to the angle domain at angle $\phi$ therefore requires an inner product with the corresponding spatial steering vector. Similarly, the temporal steering $\mathbf{b}(f)$ vector corresponding to a Doppler frequency $f$ is the magnitude and phase taper measured at an individual element for the $M$ pulses in a CPI. An inner product with the corresponding temporal steering vector transforms time domain data to the Doppler domain. The angle-Doppler response of the data vector $\mathbf{x}$ at angle $\phi$ and Doppler $f$ is therefore given by

$$
\tilde{x}(\phi, f)=\mathbf{v}^{H}(\phi, f) \mathbf{x}=[\mathbf{b}(f) \otimes \mathbf{a}(\phi)]^{H} \mathbf{x}
$$

where the tilde $\left(^{\sim}\right)$ above the scalar $x$ signifies the transform domain. Using Eqn. (10) and choosing a set of spatial and temporal steering vectors generates a corresponding vector of data in the transform domain. The adaptive weights associated with this LPR are determined using Eqns. (7) and (8) with an angle-Doppler covariance matrix $\tilde{\mathbf{R}}$ estimated from angle-Doppler secondary data. The steering vector used $(\tilde{\mathbf{v}})$ is the space-time steering vector transformed to the angle-Doppler domain.

Equations (3) and (4) show that, assuming a linear equispaced array of point sensors, the spatial and temporal steering vectors both form Fourier coefficients. For an ideal array the JDL processor can be simplified under the following two conditions. In the original development of [14], the authors assume both these conditions are met.

1. If a set of angles are chosen such that $\left(\frac{d}{\lambda} \sin \phi\right)$ is spaced by $1 / N$ and a set of Doppler frequencies are chosen such that $\left(f / f_{R}\right)$ is spaced by $1 / M$, the transformation to the angleDoppler domain is equivalent to the 2D-DFT, an orthogonal transformation.

2. If the look angle $\phi_{t}$ corresponds to one of these angles and the look Doppler $f_{t}$ corresponds to one of these Dopplers, the space-time steering vector is a column of the 2D-DFT matrix and the angle-Doppler steering vector is localized to a single angle-Doppler bin, i.e.,

$$
\tilde{\mathbf{v}}=[0,0, \cdots, 0,1,0, \cdots, 0,0]^{T} .
$$

In practice it is impossible to meet these conditions. The elements of a real array must be some non-zero size. This implies the elements not only sample the incident fields, but also reradiate them, leading to mutual coupling between array elements. The spatial steering vector of Eqn. (3) is not valid and a DFT does not transform spatial data to the angle domain. A mismatch between the spatial channels leads to a similar effect, as does the use of an array without linear, equi-spaced, elements.. A DFT is mathematically valid, but has no physical meaning. Even in the case of simulations based on ideal linear arrays, the use of a 2D-DFT restricts the spacing between the angle and Doppler bins as the 2D-DFT can form only $N$ orthogonal spatial beams and $M$ orthogonal temporal beams. These restrictions are unnecessary since the approach in [5] can account for any non-orthogonality in the spatio-temporal beams. 
The basis of the new approach is that the transformation to the angle-Doppler domain, as described by Eqn. (10), is valid for both ideal linear arrays and real world arrays. In the case of a real array, the true spatial steering vector must be used for the transformation. This steering vector must be either measured or obtained using a numerical electromagnetic analysis. The measured steering vector would also account for channel mismatch.

In the JDL algorithm, only data from within the LPR is used for the adaptation process. Equation (10) indicates that the transformation from the space-time domain to the angle-Doppler domain is, in effect, an inner product with a space-time steering vector. The relevant transformation to the data within the LPR is

$$
\tilde{\mathbf{x}}_{\mathrm{LPR}}=\mathbf{T}^{H} \mathbf{x}
$$

where $\mathbf{T}$ is a $\left(N M \times \eta_{a} \eta_{d}\right)$ transformation matrix. For example, based on Eqn. (10), if the LPR covers three angle bins $\left(\phi_{-1}, \phi_{0}, \phi_{1} ; \eta_{a}=3\right)$ and three Doppler bins $\left(f_{-1}, f_{0}, f_{1} ; \eta_{d}=3\right)$

$$
\begin{gathered}
\mathbf{T}=\left[\begin{array}{ccc}
\mathbf{b}\left(f_{-1}\right) \otimes \mathbf{a}\left(\phi_{-1}\right) & \mathbf{b}\left(f_{-1}\right) \otimes \mathbf{a}\left(\phi_{0}\right) & \mathbf{b}\left(f_{-1}\right) \otimes \mathbf{a}\left(\phi_{1}\right) \\
\mathbf{b}\left(f_{0}\right) \otimes \mathbf{a}\left(\phi_{-1}\right) & \mathbf{b}\left(f_{0}\right) \otimes \mathbf{a}\left(\phi_{0}\right) & \mathbf{b}\left(f_{0}\right) \otimes \mathbf{a}\left(\phi_{1}\right) \\
\mathbf{b}\left(f_{1}\right) \otimes \mathbf{a}\left(\phi_{-1}\right) & \mathbf{b}\left(f_{1}\right) \otimes \mathbf{a}\left(\phi_{0}\right) & \mathbf{b}\left(f_{1}\right) \otimes \mathbf{a}\left(\phi_{1}\right)
\end{array}\right] \\
=\left[\begin{array}{lll}
\mathbf{b}\left(f_{-1}\right) & \mathbf{b}\left(f_{0}\right) & \mathbf{b}\left(f_{1}\right)
\end{array}\right] \otimes\left[\begin{array}{lll}
\mathbf{a}\left(\phi_{-1}\right) & \mathbf{a}\left(\phi_{0}\right) & \mathbf{a}\left(\phi_{1}\right)
\end{array}\right]
\end{gathered}
$$

i.e., the transformation matrix is composed of steering vectors corresponding to $\eta_{a}$ angles and $\eta_{d}$ Doppler frequencies. Here the spatial steering vectors $\mathbf{a}(\phi)$ include electromagnetic effects such as mutual coupling. The angles can be chosen without restriction, usually based on antenna measurements available. Because the mutual coupling and the lack of restrictions on the angles, the columns of this transformation matrix are not orthogonal to each other, i.e., the transformation is not orthogonal.

The transformation matrix formulation also removes another restriction placed on the original algorithm. In [14], to achieve the simple form of the angle-Doppler steering vector given by Eqn. (11), the use of a low sidelobe window to lower the transform sidelobes is discouraged. The use of such a window may be easily incorporated by modifying the transformation matrix of Eqn. (14). If a length $N$ taper $\mathbf{t}_{s}$ is to be used in the spatial domain and a length $M$ taper $\mathbf{t}_{t}$ in the temporal domain, the transformation matrix is given by

$$
\mathbf{T}=\left[\mathbf{t}_{t} \odot \mathbf{b}\left(f_{-1}\right) \quad \mathbf{t}_{t} \odot \mathbf{b}\left(f_{0}\right) \quad \mathbf{t}_{t} \odot \mathbf{b}\left(f_{1}\right)\right] \otimes\left[\mathbf{t}_{s} \odot \mathbf{a}\left(\phi_{-1}\right) \quad \mathbf{t}_{s} \odot \mathbf{a}\left(\phi_{0}\right) \quad \mathbf{t}_{s} \odot \mathbf{a}\left(\phi_{1}\right)\right]
$$

where $\odot$ represents the Hadamard product, a point-by-point multiplication of two vectors.

In the modified JDL formulation, the angle-Doppler steering vector used to solve for the adaptive weights in Eqn. (7) is the space-time steering vector $\mathbf{v}$ transformed to the angle-Doppler domain 
via the same transformation matrix $\mathbf{T}$, i.e.,

$$
\tilde{\mathbf{v}}=\mathbf{T}^{H} \mathbf{v} .
$$

Note that the non-orthogonal transformation results in spreading of the target signal in angle. Unlike the case of Eqn. (11), the angle-Doppler steering vector has more than one non-zero entry. The use of a low sidelobe window, as in Eqn. (15), causes spreading in both angle and Doppler.

\subsection{Non-homogeneity Detection}

Assuming array effects have been accounted for, the first step in a practical STAP algorithm is a non-homogeneity detector to determine which sections of the received data are non-homogeneous. Non-homogeneities may be distributed or localized. Distributed clutter non-homogeneities occur when two or more kinds of terrain are illuminated, for example at a land-sea interface. Discretes may arise in urban and spiky clutter scenarios due to natural and man-made features or be caused by large targets in the transmit sidelobes. A target-like signal in the primary range cell, but not at the look angle-Doppler, is effectively discrete interference. This situation may arise due to a targetlike signal in the sidelobe due to a moving vehicle in the sidelobe but within the primary range cell, a corner reflector or a coherent jammer. True targets (targets at the look angle-Doppler) must also be considered discrete interference when the range bin containing the target is a secondary data sample for other primary range cells.

Statistical algorithms suffer when the secondary data is non-homogeneous, i.e., the data does not reflect the interference statistics in the primary range cell. There is no unique definition of what makes a range cell non-homogeneous and researchers have relied on an engineering definition. To quote [15], "A data set is termed wide sense homogeneous if the system performance loss can be ignored or is acceptable for a given STAP algorithm. A data set is said to be wide-sense nonhomogeneous if it is not wide-sense homogeneous".

In keeping with this definition of non-homogeneous data, we use here a NHD technique that uses the JDL-MSMI statistic as an indication of a non-homogeneity [4]. Other forms of the NHD, such as the Adaptive Power Residue (APR) [11] and Generalized Inner Product (GIP) [16], are not considered here. In a homogeneous data scenario, the STAP process suppresses interference and the detection statistic corresponds to thermal noise. Any significant deviations are due to non-homogeneities, since even true targets are effectively non-homogeneities. The NHD used here is therefore exactly the same process as detecting targets in a homogeneous scene. The NHD applies the JDL algorithm, assuming all available data is homogeneous, for the chosen look angle and Doppler to yield a MSMI statistic. Any range cell with a statistic above a chosen threshold is considered non-homogeneous. Only those non-homogeneities that impact the STAP algorithm are 
identified by this process. A particular range may be declared non-homogeneous for a particular Doppler bin and not for another.

Having separated the data cube into homogeneous and non-homogeneous range cells, a statistical algorithm such as JDL is appropriate within the homogeneous cells. The difference is that only other homogeneous cells are used as secondary data to estimate the covariance matrix.

\subsection{Hybrid Processing in Non-homogeneous Range Cells}

A range cell may be deemed non-homogeneous by the NHD due to a target, a strong discrete in the sidelobes or due to a poor estimate of the covariance matrix leading to a false alarm. Secondary data cells do not carry information about the non-homogeneity and hence a purely statistical algorithm cannot suppress uncorrelated interference within the range cell under test.

The inability of statistical STAP algorithms to counter non-homogeneities in the primary data motivates research in the area of non-statistical or direct data domain $\left(\mathrm{D}^{3}\right)$ algorithms. These algorithms use data from the range cell of interest only, eliminating the need for secondary data support associated with statistical approaches. Researchers have mainly focused on one-dimensional spatial adaptivity [17-19]. This paper extends the algorithm of [19] to formulate a two-dimensional $\mathrm{D}^{3}$ space-time algorithm [6].

\subsubsection{Two Dimensional Direct Data Domain Processing}

To best present the $\mathrm{D}^{3}$ algorithm, we work with $\mathbf{X}_{n m}$, the data at the $n$-th element and $m$-th pulse of the $N \times M$ data matrix $\mathbf{X}$ from the primary range cell only. The algorithm is presented here for the ideal case of a uniformly spaced linear array of isotropic sensors. Compensating for mutual coupling and channel mismatch is described in context in Section 3.1.1. Using the signal phase progression in Eqns. (5) and (6), the signal component is eliminated from terms such as $\left(\mathbf{X}_{n m}-z_{s} \mathbf{X}_{(n+1) m}\right)$ and $\left(\mathbf{X}_{n m}-z_{t} \mathbf{X}_{n(m+1)}\right)$, leaving only residual interference terms. $\mathrm{D}^{3}$ methods use this fact to obtain adaptive weights that minimize the residual interference power within the primary range cell. Define the matrix $\mathbf{B}$ to be

$$
\mathbf{B}=\left[\begin{array}{cccc}
\mathbf{X}_{00}-z_{t} \mathbf{X}_{01} & \mathbf{X}_{01}-z_{t} \mathbf{X}_{02} & \cdots & \mathbf{X}_{0(M-2)}-z_{t} \mathbf{X}_{0(M-1)} \\
\mathbf{X}_{10}-z_{t} \mathbf{X}_{11} & \mathbf{X}_{11}-z_{t} \mathbf{X}_{12} & \cdots & \mathbf{X}_{1(M-2)}-z_{t} \mathbf{X}_{1(M-1)} \\
\vdots & \vdots & \vdots & \vdots \\
\mathbf{X}_{(N-1) 0}-z_{t} \mathbf{X}_{(N-1) 1} & \mathbf{X}_{(N-1) 1}-z_{t} \mathbf{X}_{(N-1) 2} & \cdots & \mathbf{X}_{(N-1)(M-2)}-z_{t} \mathbf{X}_{(N-1)(M-1)}
\end{array}\right]
$$

Theoretically, the entries of the $N \times(M-1)$ matrix $\mathbf{B}$ carry interference terms only, but due to beam mismatch, there is some residual target information in the entries of $\mathbf{B}$. However, unless the 
target is significantly off the look direction/Doppler, the target signal is effectively nulled in the entries of this matrix. In the case where the target is significantly off the look direction, it must be treated as discrete interference; in a surveillance radar, targets must be declared only if they are within a beamwidth of the look direction. In fact, sidelobe targets are an example of the discrete interference driving this research. The overall $\mathrm{D}^{3}$ algorithm does not null mainlobe targets [6].

Consider the following scalar functions of a set of temporal weights $\mathbf{w}_{t}$,

$$
\begin{aligned}
G_{\mathbf{w}_{t}} & =\left|\mathbf{b}_{(0: M-2)}^{H} \mathbf{w}_{t}\right|^{2}=\mathbf{w}_{t}^{H} \mathbf{b}_{(0: M-2)} \mathbf{b}_{(0: M-2)}^{H} \mathbf{w}_{t}, \\
I_{\mathbf{w}_{t}} & =\left\|\mathbf{B}^{*} \mathbf{w}_{t}\right\|_{2}^{2}=\mathbf{w}_{t}^{H} \mathbf{B}^{T} \mathbf{B}^{*} \mathbf{w}_{t}, \\
R_{\mathbf{w}_{t}} & =G_{\mathbf{w}_{t}}-\kappa^{2} I_{\mathbf{w}_{t}},
\end{aligned}
$$

where $^{*}$ represents the complex conjugate, $\|\cdot\|_{2}$ the 2 -norm of a vector and $\mathbf{b}_{(0: M-2)}$ the first $(M-1)$ entries of the temporal steering vector defined by Eqn. (4). In Eqn. (19), $\mathbf{B}^{*} \mathbf{w}_{t}$ is used to remain consistent with the term $\mathbf{b}_{(0: M-2)}^{H} \mathbf{w}_{t}$, in that the weights multiply the conjugate of the data [6].

The term $G_{\mathbf{w}_{t}}$ in Eqn. (18) represents the gain of the weight vector $\mathbf{w}_{t}$ at the look Doppler frequency $f_{t}$ while the term $I_{\mathbf{w}_{t}}$ in Eqn. (19) represents the residual interference power after the data is filtered by the same weights. Hence, $R_{\mathbf{w}_{t}}$ in Eqn. (20) represents the difference between the gain of the antenna at the look Doppler and the residual interference power. The $\mathrm{D}^{3}$ algorithm finds the weight vector that maximizes this difference. Mathematically,

$$
\max _{\left\|\mathbf{w}_{t}\right\|_{2}=1} R_{\mathbf{w}_{t}}=\max _{\left\|\mathbf{w}_{t}\right\|_{2}=1}\left[G_{\mathbf{w}_{t}}-\kappa^{2} I_{\mathbf{w}_{t}}\right]=\max _{\left\|\mathbf{w}_{t}\right\|_{2}=1} \mathbf{w}_{t}^{H}\left[\mathbf{b}_{(0: M-2)} \mathbf{b}_{(0: M-2)}^{H}-\kappa^{2} \mathbf{B}^{T} \mathbf{B}^{*}\right] \mathbf{w}_{t}
$$

where the constraint $\left\|\mathbf{w}_{t}\right\|_{2}=1$ is chosen to obtain a finite solution and $\kappa$ represents a trade off between mainbeam gain and interference suppression. Using the method of Lagrange multipliers, it can be shown that the desired temporal weight vector is the eigenvector corresponding to the maximum eigenvalue of the $(M-1) \times(M-1)$ matrix $\left[\mathbf{b}_{(0: M-2)} \mathbf{b}_{(0: M-2)}^{H}-\kappa^{2} \mathbf{B}^{T} \mathbf{B}^{*}\right]$. This formulation yields a temporal weight vector of length $(M-1)$.

The spatial weight vector $\mathbf{w}_{s}$ can be found in an analogous manner. The length $N M$ space-time adaptive weight vector, for look angle $\phi_{t}$ and look Doppler $f_{t}$, is then given by

$$
\mathbf{w}\left(\phi_{t}, f_{t}\right)=\left[\begin{array}{c}
\mathbf{w}_{t} \\
0
\end{array}\right] \otimes\left[\begin{array}{c}
\mathbf{w}_{s} \\
0
\end{array}\right] .
$$

A single degree of freedom is used to eliminate the target component in the entries of the matrix B. The zeros appended to the spatial and temporal weight vectors represent this one degree of freedom lost. 


\subsubsection{Two Stage Hybrid Algorithm}

Within a non-homogeneous range cell, interference has both discrete and correlated components. The $\mathrm{D}^{3}$ approach is effective in countering discrete non-homogeneities within the primary range cell. However, by using data from within the primary range cell only, $\mathrm{D}^{3}$ techniques ignore all correlation information and are not as effective against correlated interference. The key to STAP within nonhomogeneous range cells is to combine the benefits of $\mathrm{D}^{3}$ and statistical adaptive processing. This hybrid approach uses the $\mathrm{D}^{3}$ algorithm above as a first stage adaptive transform to the angleDoppler domain, i.e., the $\mathrm{D}^{3}$ algorithm replaces the non-adaptive transform used in Section 2.2. A second stage of statistical JDL processing in the angle-Doppler domain follows. The hybrid algorithm is therefore a cascade of the $\mathrm{D}^{3}$ and JDL algorithms, combining the benefits of both.

Consider the general framework of any STAP algorithm. The algorithm processes received data to obtain a complex weight vector for each range bin and each look angle/Doppler. The weight vector then multiplies the primary data vector to yield a complex number. Obtaining a real scalar from this number for threshold comparison is part of the post-processing and not inherent to the algorithm itself. Therefore, STAP obtains an adaptive estimate of the signal component in the look direction. The adaptive weights can be viewed in a role similar to the non-adaptive steering vectors, used in Section 2.2 to transform the space-time data to the angle-Doppler domain.

Section 2.2 represents the transformation process as a multiplication with a transformation matrix T. By choosing the set of look angles and Dopplers to be points in the LPR, the $\mathrm{D}^{3}$ weights perform a function analogous to the non-adaptive transform. The hybrid approach uses the $\mathrm{D}^{3}$ weights as the columns of this matrix, replacing the non-adaptive steering vectors used earlier. Statistical JDL processing in the angle-Doppler domain then continues as before. Using the $\mathrm{D}^{3}$ weights from Eqn. (22), the transformation matrix for the LPR covering three angle bins $\left(\phi_{-1}, \phi_{0}, \phi_{1} ; \eta_{a}=3\right)$ and three Doppler bins $\left(f_{-1}, f_{0}, f_{1} ; \eta_{d}=3\right)$ is now given by

$$
\begin{aligned}
& \mathbf{T}=\left[\mathbf{w}\left(\phi_{-1}, f_{-1}\right) \mathbf{w}\left(\phi_{0}, f_{-1}\right) \mathbf{w}\left(\phi_{1}, f_{-1}\right)\right. \\
& \mathbf{w}\left(\phi_{-1}, f_{0}\right) \quad \mathbf{w}\left(\phi_{0}, f_{0}\right) \quad \mathbf{w}\left(\phi_{1}, f_{0}\right) \\
& \left.\mathbf{w}\left(\phi_{-1}, f_{1}\right) \mathbf{w}\left(\phi_{0}, f_{1}\right) \mathbf{w}\left(\phi_{1}, f_{1}\right)\right] .
\end{aligned}
$$

This transformation matrix is also used to transform the secondary data to the angle-Doppler domain and to obtain the angle-Doppler steering vector, as in Eqn. (16). Advantages associated with the JDL algorithm, such as the reduced required secondary data support, carry over to the hybrid algorithm. However, unlike the JDL algorithm this transformation matrix changes from range cell to range cell and must be evaluated for each range cell deemed non-homogeneous. This makes the hybrid algorithm significantly more computationally intensive. To minimize the computation load it must be used only as necessary. 
In testing using simulations and measured data the hybrid algorithm has shown the ability to suppress both discrete and correlated interference [6]. This is a significant advance over both traditional statistical processing techniques, such as JDL, and $\mathrm{D}^{3}$ approaches.

This development of the hybrid algorithm completes the presentation of each component of the KB-STAP approach of Fig. 1. The next section puts these individual components together into a comprehensive, practical, approach to STAP for GMTI.

\section{Knowledge Based Adaptive Processing}

Knowledge based processing best matches the adaptive processing algorithm to the interference scenario. The algorithm is chosen using knowledge gained by processing the received data. In the KB-processor of Fig. 1, the data is classified into one of only two types: homogeneous or nonhomogeneous, with different algorithms used for each type of data. This classification is made using the NHD of Section 2.3 based on whether the JDL-MSMI statistic crosses a chosen threshold. Section 3.2 discusses extensions to this simple KB-processor, including other information sources that may inform the knowledge base.

Within the range cells deemed non-homogeneous, the interference is assumed to have discrete and homogeneous components and the hybrid algorithm is used for target detection. While theoretically there is no restriction on what statistical algorithm is used for adaptive processing in the homogeneous range cells, here we use the JDL algorithm of Section 2.2, allowing for the JDL algorithm to be the basis for all three components of the KB-processor in 1 . The only difference between processing in the homogeneous cells and in the non-homogeneous cells is the choice of transformation matrix. Within the homogeneous cells, the transformation matrix is the non-adaptive transform of Eqn. (15). Within the non-homogeneous range cells, the transformation matrix is the adaptive $\mathrm{D}^{3}$ transform of Eqn. (23). In both cases, the secondary data cells used to estimate the angle-Doppler covariance matrix are chosen from range cells deemed homogeneous.

\subsection{Numerical Example}

The motivation for the KB-processor is practical implementation of STAP in airborne radars for GMTI. With this in mind, the KB formulation is tested using measured data from the MCARM program [12]. The example chosen here uses the data from acquisition 575 on flight 5. Included with the data is information regarding the position, aspect, and velocity of the airborne platform and the mainbeam transmit direction for each CPI. This information is used to correlate target detections with ground features.

While recording this acquisition, the radar platform was at latitude-longitude coordinates of 


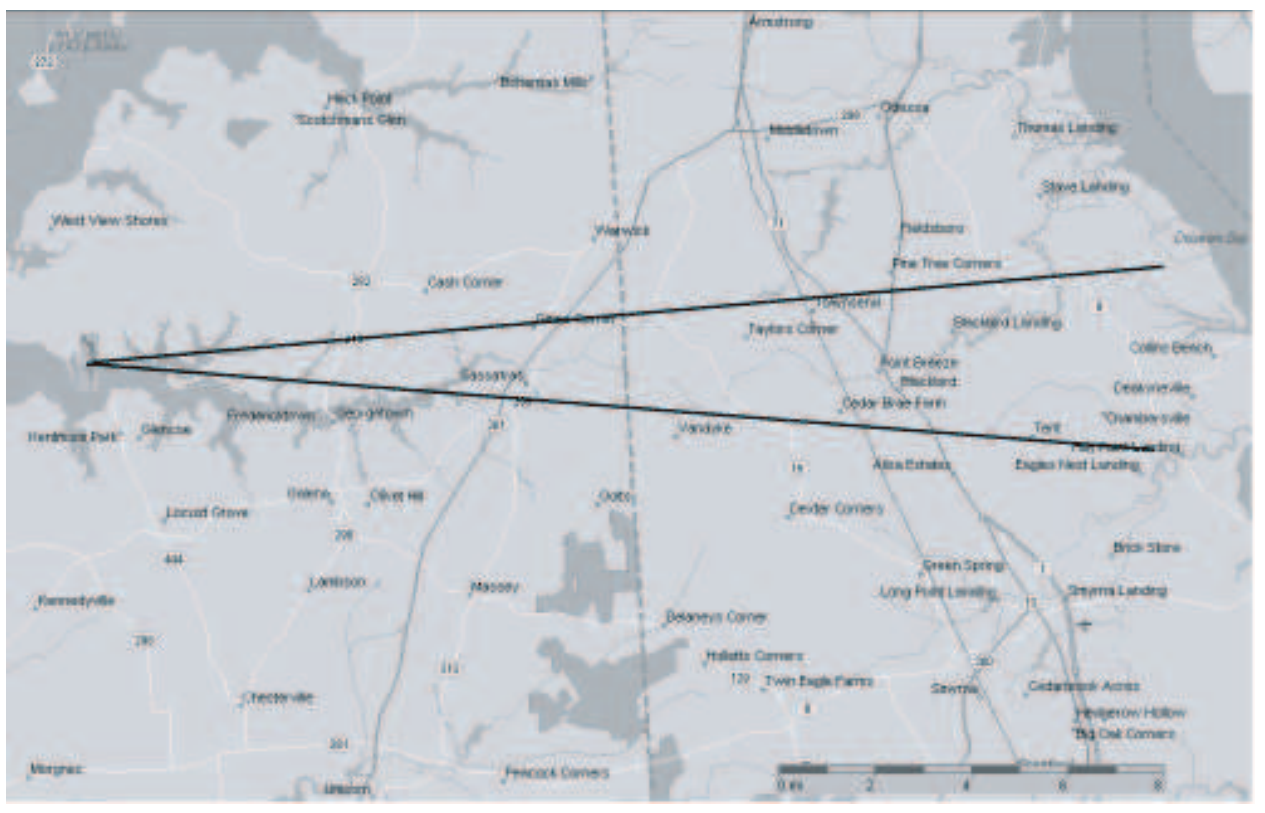

Figure 2: Location and transmit direction of the MCARM airplane during acquisition 575.

$\left(39.379^{\circ},-75.972^{\circ}\right)$, placing the aircraft close to Chesapeake Haven, Maryland, USA. The plane was flying mainly south with velocity $223.78 \mathrm{mph}$ and east with velocity $26.48 \mathrm{mph}$. The aircraft location and the transmit mainbeam are shown in Fig. 2. The mainbeam is close to broadside. Note that the mainbeam illuminates terrain of various types, including several major highways. Each CPI comprises 22 elements $(N=22), 128$ pulses $(M=128)$ at a PRF of $1984 \mathrm{~Hz}$ and 630 range bins sampled at $0.8 \mu \mathrm{s}$ (corresponding to 0.075 miles). The array is a $2 \times 11$ rectangular array. The array operates at a center frequency of $1.24 \mathrm{GHz}$.

To illustrate the effects of non-homogeneities in secondary training data we inject two targets at closely spaced range bins. These artificial targets are in addition to the ground targets of opportunity on the roadways illuminated by the array. The artificial targets are injected in range bins 290 and 295. In this acquisition, the zero range is referenced to range bin 74 and so these injected targets are at ranges of 16.2 miles and 16.575 miles respectively. The parameters of the injected targets are given in Table 1. It is difficult to determine the associated signal to noise ratio of the these targets because the noise and level in the MCARM program is unknown. These values are chosen to ensure that the targets cannot be distinguished using non-adaptive, matched filter, processing. Note that the two targets are at the same look angle and Doppler frequency and the second target is $20 \mathrm{~dB}$ stronger than the first.

This example uses three angle bins and three Doppler bins $($ a $3 \times 3 \mathrm{LPR})$ in all stages of adaptive processing, including the JDL-NHD. Thirty six secondary data vectors are used to estimate the $9 \times 9$ angle-Doppler LPR covariance matrix. Two guard cells are used on either side of the primary data vector. Based on these numbers, without a NHD stage, range bin 295 would be used as a secondary 
Table 1: Parameters defining the injected targets.

\begin{tabular}{|c|c|c|}
\hline & Target 1 & Target 2 \\
\hline Ampl & $1 \times 10^{-4}$ & $1 \times 10^{-3}$ \\
\hline Angle bin & $1^{o}$ & $1^{o}$ \\
\hline Doppler & $-9 \equiv-139.5 \mathrm{~Hz}$ & $-9 \equiv-139.5 \mathrm{~Hz}$ \\
\hline Range bin & $290 \equiv 16.2 \mathrm{mi}$ & $295 \equiv 16.575 \mathrm{mi}$ \\
\hline
\end{tabular}

data vector for detection within range bin 290, violating the i.i.d. assumption of statistical STAP algorithms. The example compares three results: from the original JDL algorithm of [14], the JDL algorithm accounting for array effects of [5] and the KB-STAP algorithm of Fig. 1.

Figure 3 plots the results of the original JDL algorithm without attempting to compensate for array effects or non-homogeneities. The plot is of the MSMI statistic as a function of range and Doppler. The red spots correspond to higher statistics, i.e., the red tend to correspond to target detections. The figure shows that targets are detected in almost all range and Doppler bins, including at extremely high velocities. If using the original JDL algorithm with measured data, therefore, one must deal with several false alarms. Also, while the second injected target is clearly visible, the first target is not detected at all. This inability to detect the target is because the second target is present in the secondary data while attempting to detect the first target at range bin 290. The presence of a target-like non-homogeneity in the secondary data makes detection of a weak target practically impossible.

Figure 4 plots the results of the JDL algorithm after accounting for real world array effects, specifically mutual coupling and channel mismatch. In comparing with Fig. 3, it is clear that the incidence of false alarms have been significantly reduced. Furthermore, the target detections stand out in better contrast from the background, i.e., there is better discrimination between target signals and residual clutter. As will be shown later, some of these target detections correspond to highways illuminated by the radar mainbeam. The improved discrimination is consistent with the results of [5], showed gains on order of several $\mathrm{dB}$ in separation between target and residual interference. However, several false alarms are still visible at extremely high velocities. In addition, the first injected target is not detected. This is because the new JDL formulation, just like the original, does not account for non-homogeneities.

\subsubsection{Implementation of the KB-Processor}

The high rates of false alarms and the inability of statistical techniques to detect a weak target in non-homogeneous interference indicates the importance of the non-homogeneity detector and the 


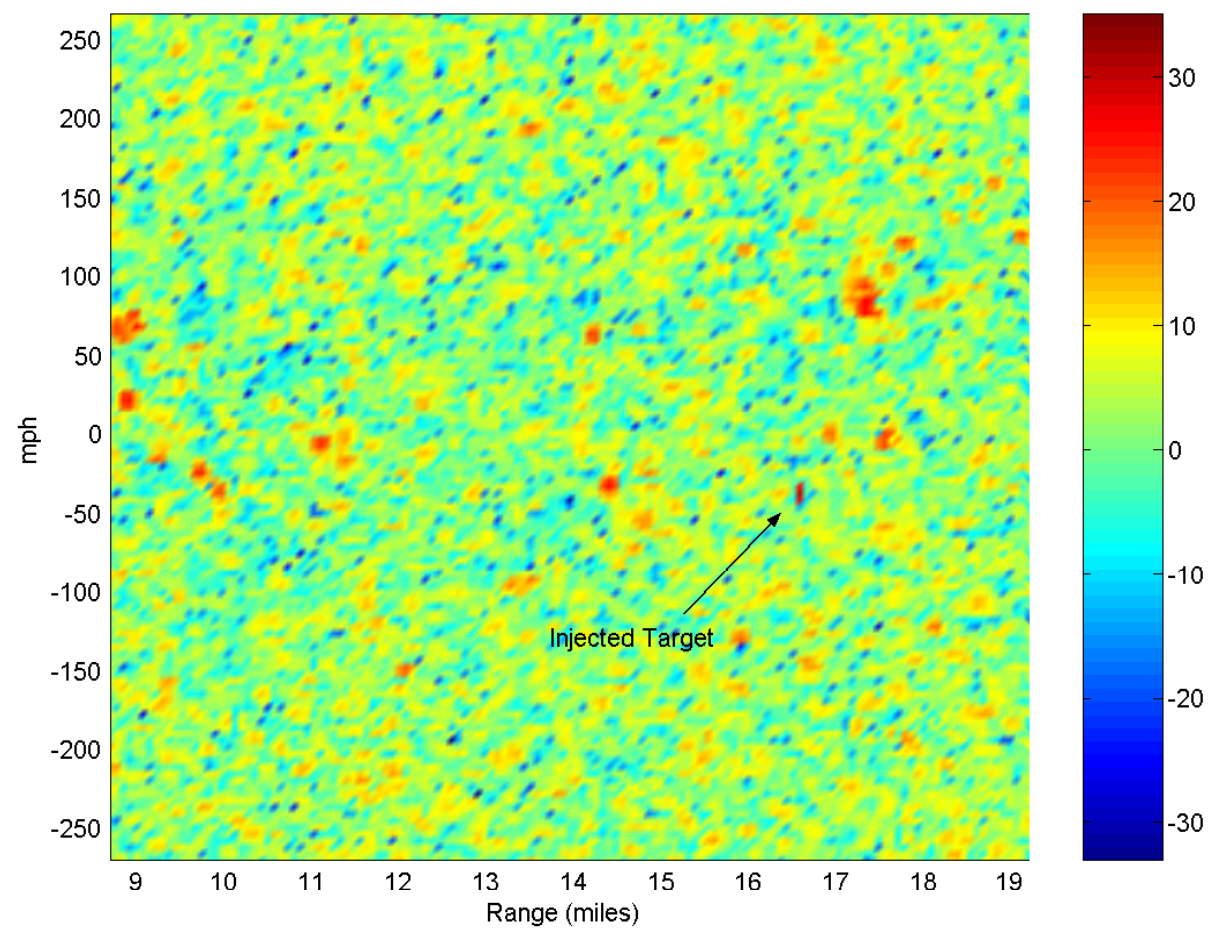

Figure 3: JDL Processing ignoring array effects and non-homogeneities.

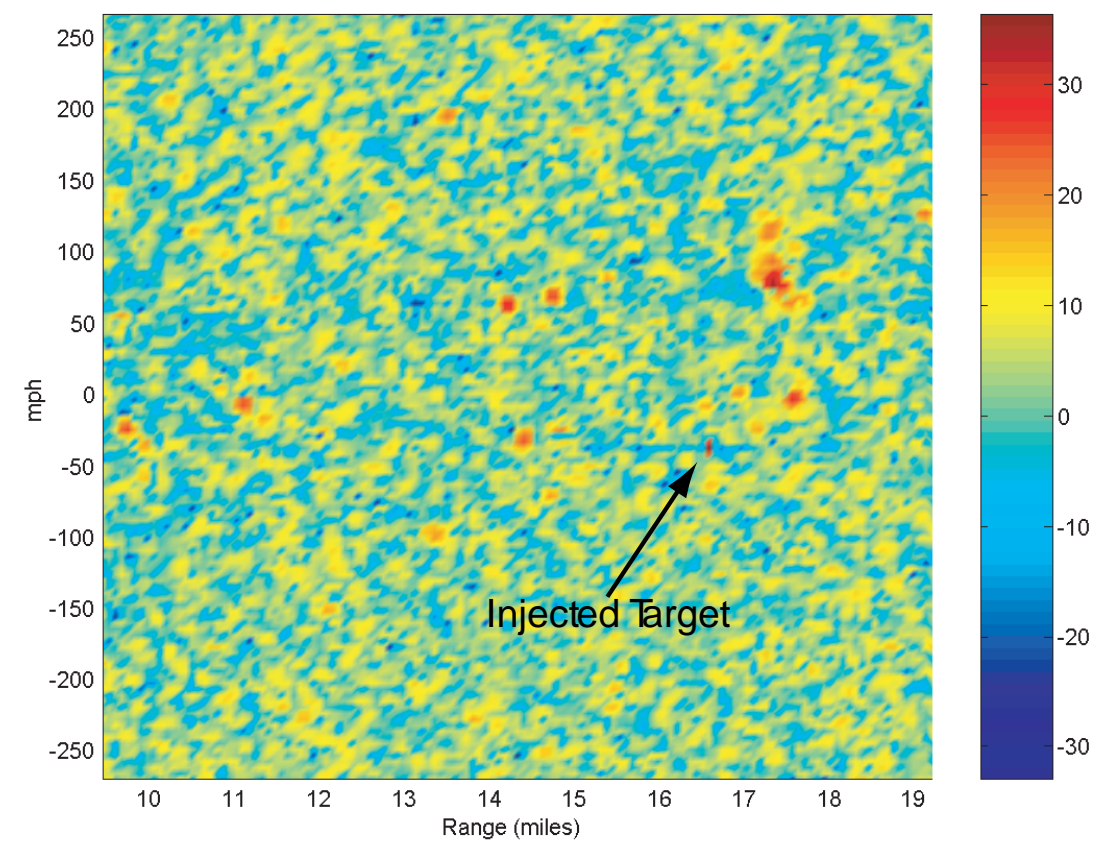

Figure 4: JDL Processing accounting for array effects, but ignoring non-homogeneities. 
need for hybrid processing in the non-homogeneous range cells. In implementing the KB-processor, certain parameters must be chosen. Furthermore, in Section 2.4.1, the $\mathrm{D}^{3}$ algorithm is developed for an ideal linear array of point sensors. The algorithm must be modified before it can be applied to the MCARM rectangular array.

The first step in the KB-processor is the NHD, here the JDL-NHD. At this stage, all range cells are used to obtain the sample support required to execute the JDL-NHD algorithm. A range cell is considered non-homogeneous if its decision statistic is above 18.52. Assuming homogeneous Gaussian interference, using 36 secondary data vectors to estimate a $9 \times 9$ covariance matrix, this threshold corresponds to a false alarm rate of $P_{f a}=0.0001^{2}$. Note that targets that fall above this threshold will also be treated as non-homogeneities. In this acquisition, approximately $5 \%$ of the test statistics fall above this threshold. If the data were truly Gaussian and homogeneous, only $0.01 \%$ of test statistics should fall above this threshold.

Within each cell deemed non-homogeneous, the hybrid algorithm is used. To be able to implement the $\mathrm{D}^{3}$ algorithm to the MCARM data we use an ad-hoc procedure to approximate an ideal, linear array. Equations (3) and (5) indicate that the spatial steering vector at broadside $(\phi=0)$ is given by $\mathbf{a}(\phi=0)=\left[\begin{array}{llll}1 & 1 & \cdots & 1\end{array}\right]^{T}$. In the absence of mutual coupling or channel mismatch, this steering vector at broadside is valid for arrays in any configuration. The approach taken here is to artificially rotate all the data, using the measured spatial steering vector, so as to force the look direction to broadside. This compensates for the rectangular array configuration and also the mutual coupling and channel mismatch associated with the look direction. The rotation is achieved by an entry-by-entry division of the received voltages at the array level with the measured spatial steering vector corresponding to the look direction. Using pseudo-MATLAB notation, this operation can be represented by

$$
\overline{\mathbf{x}}(m)=\mathbf{x}(m) \cdot / \mathbf{a}_{m}\left(\phi_{t}\right)
$$

where $\mathbf{x}(m)$ represents the $N$ returns from the $m$-th pulse in a CPI, and $\mathbf{a}_{m}\left(\phi_{t}\right)$ represents the measured steering vector corresponding to the look direction $\phi_{t}$. It is repeated for all pulses in all range bins. The division operation of Eqn. (24) forces the effective spatial steering vector for any look direction to be $\overline{\mathbf{a}}\left(\phi_{t}\right)=\left[\begin{array}{llll}1 & 1 & \cdots & 1\end{array}\right]^{T}$, equivalent to broadside in an ideal array. This operation also rotates the effective elevation angle to broadside. The $\mathrm{D}^{3}$ method of Section 2.4.1 is applied to the "rotated" data $\overline{\mathbf{x}}$ with broadside as the look direction.

The KB-processor, illustrated in Fig. 1, matches the processing to the interference in that it uses JDL processing in the homogeneous range cells and hybrid processing in the non-homogeneous cells. In both cases, a $3 \times 3 \mathrm{LPR}$ is used. In the second application of the JDL algorithm within homogeneous range cells, only other homogeneous cells, identified by the JDL-NHD, are used for

\footnotetext{
${ }^{2}$ The authors would like to thank Dr. Yuhong Zhang of Stiefvater Consultants, Marcy, NY, for providing with the false alarm rate used in this paper.
} 


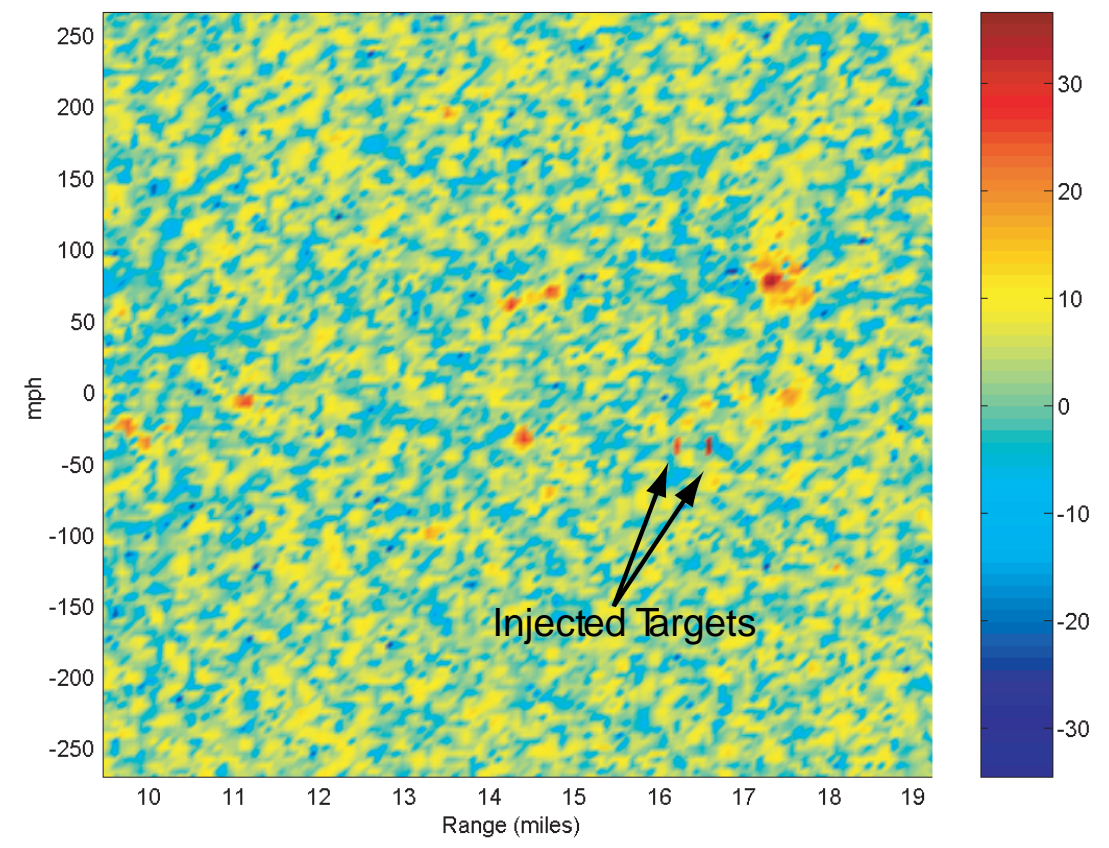

Figure 5: KB-processor matching the STAP algorithm to the interference scenario.

sample support. Within each non-homogeneous cell, the hybrid algorithm is used. This requires $\mathrm{D}^{3}$ algorithm be applied a total of nine times corresponding to the three angle and three Doppler look directions, using the same primary data. The second stage in the hybrid algorithm is JDL processing of the angle-Doppler data. The angle-Doppler data obtained using the $\mathrm{D}^{3}$ processing is used for in this second stage JDL processing. This requires estimation of an interference covariance matrix. Homogeneous cells, identified by the NHD stage, are used to obtain sample support for the second stage JDL process.

Figure 5 plots the MSMI statistic obtained by using the KB-processor. The improved discrimination, as compared to Figs. 3 and 4 between a few target signals and residual interference is clear. The first target is now clearly visible. This is possible because the NHD treats the second injected target as a non-homogeneity and it is eliminated from the secondary data while processing the range cell corresponding to the first, weaker, injected target. The KB-processor can, therefore, detect weak targets buried in non-homogeneous interference.

The final step in determining the presence or absence of a target is to apply a threshold to the MSMI statistic of Figs. 3 and 5 to yield target declarations. Here, a target is declared at all points with a MSMI statistic of greater than 40. Figures 6 and 7 plot the declared target locations as a function of Doppler and range. These locations are correlated with the map of Fig. 2. In Fig. 6, note the extremely high number of false alarms. Also, as in Fig. 3, the weak injected target is not detected. On the other hand, nearly all the target declarations by the KB-processor, in 


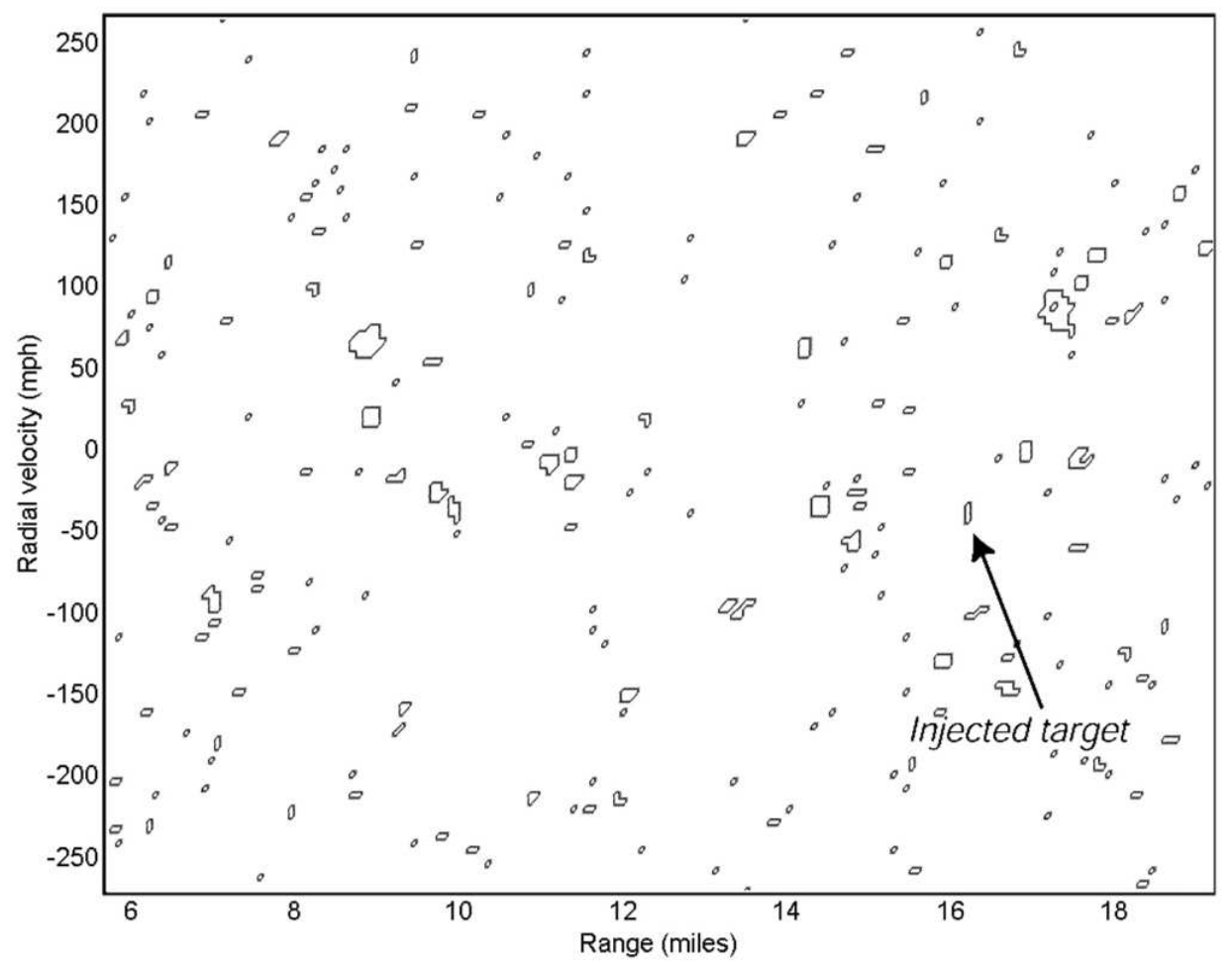

Figure 6: Target declarations using JDL ignoring array effects and non-homogeneities.

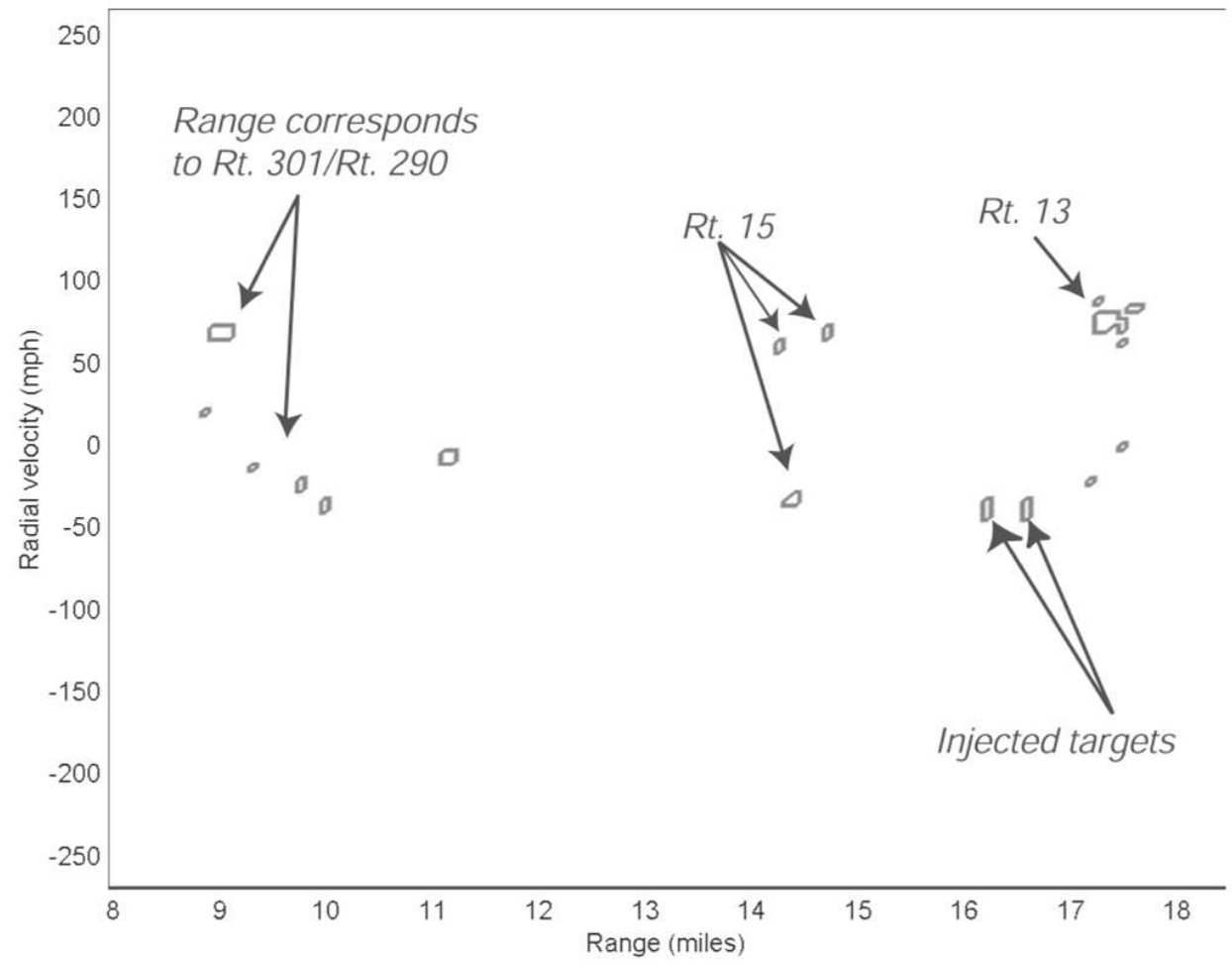

Figure 7: Target declarations using Knowledge Based STAP. 
Fig. 7, correlate directly with major highways in Maryland and Delaware illuminated by the radar mainbeam. Routes 290 and 301 in Maryland are closely spaced at a range of 9.0 and 9.8 miles. Accounting for the platform motion, the ground speed of the target(s) is approximately $50 \mathrm{mph}$.

In essence the hybrid algorithm has been applied to all range/Doppler bins where the JDL-MSMI statistic is greater than 18.52. The hybrid algorithm suppresses non-homogeneities, significantly reducing false alarms. In addition, the weaker injected target is detected because the stronger target at range bin 295 is eliminated from the sample support. Note that because the NHD does not identify this range bin (range bin 290) as a non-homogeneity, the weak target is detected using statistical JDL processing only because other homogeneous cells are used for sample support.

The target detections at the far range shown in the plot are between 19.4 and 20.4 miles. The range to Route 9 varies between 19.1 and 21.1 miles within the transmit mainbeam. These far range detections therefore correspond to Route 9 . The targets detected at these ranges are present in both Figs. 6 and 7.

\subsection{Potential Enhancements to KB-STAP}

The KB-processor presented here is the simplest formulation where the adaptive process is chosen to match the interference within the primary range cell. The choice of the JDL-NHD and JDL as the statistical algorithm is only one of several possible choices. This choice is made because of the ease in implementation with all components dependent on the JDL algorithm. A more sophisticated processor would choose from several possible statistical algorithms to maximize the probability of detection for a given false alarm rate. For example, the choice could be from with the algorithm presented here and the knowledge-based algorithms in [8-11].

The key difference between any KB-processor and traditional processing is the use of a knowl-

edge base. There are several sources that may inform this knowledge. As described by Antonik et al. [20], some of the possible information sources are: elevation and cover data to distinguish different terrains using a-priori map information so only secondary data from similar terrain is used, other sensors of opportunity to key in on likely target locations, feedback from other stages such as the tracker, information from other passes or flights to locate discrete non-homogeneities, etc.

\section{Conclusions}

This paper furthers the development of knowledge-based adaptive processing for ground moving target indication. Traditional statistical processing techniques are inadequate in real world situations due to array effects and non-homogeneous data. The theoretical developments presented here 
are motivated by the need to apply STAP for GMTI in real world processors. This paper introduces a KB-STAP algorithm where the adaptive process is chosen to best match the interference at hand. The overall KB-STAP algorithm is based on STAP algorithms that account for real-world effects such as mutual coupling between antenna elements. The result is a practical, comprehensive, approach that addresses both theoretical and practical issues in implementing STAP for GMTI.

The development of the KB-processor required theoretical investigations into various aspects of adaptive processing in real world arrays. The three essential topics addressed here are the use of practical antenna arrays, the detection of non-homogeneous data, and adaptive processing within the non-homogeneous cells. Though these issues have been discussed elsewhere, they are presented here individually for completeness. They are then brought together into a single KB-processor.

Figure 1 represents only the essential elements of such a processor. This formulation is one starting point for future improvements to the knowledge based processing concept. The significant processing gains achievable using KB-processing drives interest in this relatively new area of research.

\section{References}

[1] J. Ward, "Space-time adaptive processing for airborne radar," Tech. Rep. F19628-95-C-0002, MIT Linclon Laboratory, December 1994.

[2] L. Brennan and I. Reed, "Theory of adaptive radar," IEEE Transactions on Aerospace and Electronic Systems, vol. 9, pp. 237-252, March 1973.

[3] I. S. Reed, J. Mallett, and L. Brennan, "Rapid convergence rate in adaptive arrays," IEEE Transactions on Aerospace and Electronic Systems, vol. 10, No. 6, pp. 853-863, Nov. 1974.

[4] R. S. Adve, T. B. Hale, and M. C. Wicks, "Transform domain localized processing using measured steering vectors and non-homogeneity detection," in Proceedings of the 1999 IEEE National Radar Conference, pp. 285-290, Apr. 1999. Boston, MA.

[5] R. S. Adve, T. B. Hale, and M. C. Wicks, "Joint domain localized adaptive processing in homogeneous and non-homogeneous environments. part I: Homogeneous environments," IEE Proceedings on Radar Sonar and Navigation, vol. 147, pp. 57-65, April 2000.

[6] R. S. Adve, T. B. Hale, and M. C. Wicks, "Joint domain localized adaptive processing in homogeneous and non-homogeneous environments. part II: Non-homogeneous environments," IEE Proceedings on Radar Sonar and Navigation, vol. 147, pp. 66-73, April 2000.

[7] D. E. Kreithen, N. B. Pulsone, C. M. Rader, and G. E. Schrader, "The MIT Lincoln Laboratory KASSPER algorithm testbed and baseline algorithm suite," in Proc. of 2002 Sensor Array and Multichannel Sig. Proc. Workshop, Aug. 2002.

[8] W. L. Melvin, G. A. Showman, and J. R. Guerci, "A knowledge-aided GMTI detection architecture," in Proc. of 2004 IEEE Radar Conference, Apr. 2004. Philadelphia. 
[9] J. S. Bergin, C. M. Teixeira, P. M. Techau, and J. R. Guerci, "STAP with knowledge-aided data pre-whitening," in Proc. of 2004 IEEE Radar Conference, Apr. 2004. Philadelphia.

[10] M. Rangaswamy, F. C. Lin, and K. R. Gerlach, "Robust adaptive signal processing methods for heterogeneous radar clutter scenarios," Signal Proc., vol. 84, pp. 1653-1665, Sept. 2004.

[11] S. Blunt, K. R. Gerlach, and M. Rangaswamy, "The enhanced FRACTA algorithm with knowledge aided covariance estimation," in Proc. of 2004 Sensor Array and Multichannel Sig. Proc. Workshop, July 2004.

[12] D. Sloper, D. Fenner, J. Arntz, and E. Fogle, "Multi-channel airborne radar measurement (MCARM), MCARM flight test," Contract F30602-92-C-0161, Westinghouse Electronic Systems, April 1996. Additional information available at http://sunrise.deepthought.rl.af.mil.

[13] L. Cai and H. Wang, "Performance comparisons of modified SMI and GLR algorithms," IEEE Transactions on Aerospace and Electronic Systems, vol. 27, No. 2, pp. 487-491, Apr. 1991.

[14] H. Wang and L. Cai, "On adaptive spatial-temporal processing for airborne surveillance radar systems," IEEE Transactions on Aerospace and Electronic Systems, vol. 30, pp. 660-699, July 1994.

[15] H.-H. Chang, Improving Space-Time Adaptive Processing (STAP) Radar Performance in Nonhomogeneous Clutter. PhD thesis, Syracuse University, August 1997.

[16] M. C. Wicks, W. L. Melvin, and P. Chen, "An efficient architecture for nonhomogeneity detection in space-time adaptive processing airborne early warning radar," in Proceedings of the 1997 International Radar Conference, pp. 295-299, October 1997. Edinburgh, UK.

[17] T. K. Sarkar and N. Sangruji, "An adaptive nulling system for a narrow-band signal with a look-direction constraint utilizing the conjugate gradient method," IEEE Transactions on Antennas and Propagation, vol. 37, pp. 940-944, July 1989.

[18] S. Park and T. K. Sarkar, "A deterministic eigenvalue approach to space time adaptive processing," in Proceedings of the IEEE Antennas and Propagation Society International Symposium, pp. 1168-1171, July 1996.

[19] T. K. Sarkar, S. Nagaraja, and M. C. Wicks, "A deterministic direct data domain approach to signal estimation utilizing non-uniform and uniform 2-d arrays," Digital Signal Processing, vol. 8, pp. 114-125, April 1998.

[20] P. Antonik, M. C. Wicks, and Y. Salama, "Knowledge based radar signal and data processing," in Proceedings of the 5th International Conference on Radar Systems, May 1999. Brest, France. 